\title{
ANISOTROPY OF THE MECHANICAL PROPERTIES OF AUSTENITIC STEEL PRODUCTS OBTAINED BY SELECTIVE LASER MELTING
}

\author{
M. G. Isaenkova ${ }^{1}$, Yu. A. Perlovich ${ }^{1}$, A. E. Rubanov ${ }^{1}$, A. V. Yudin ${ }^{1}$ \\ ${ }^{1}$ National Research Nuclear University “MEPhI”, Moscow, Russia
}

E-mail: isamarg@mail.ru; toly.rubanov@yandex.ru

\section{AUTHOR'S INFO \\ M. G. Isaenkova, Dr. \\ Phys.-Math., Prof., \\ Yu. A.Perlovich, Dr. Phys.- \\ Math., Prof., Leading \\ Scientific Researcher; \\ A. E. Rubanov, Post- \\ graduate, \\ A. V. Yudin, Post-graduate}

\section{Key words: \\ selective laser melting, austenitic steel, crystallo- graphic texture, mechani- cal properties, anisotropy, modeling, DAMASK}

\begin{abstract}
A B S T R ACT
This research looked at specimens made of austenitic steel 316L by Selective Laser Melting (SLM) and designed for tensile testing in different directions (in relation to the growth direction during printing). A significant anisotropy of mechanical properties was found as a result of mechanical testing: anisotropy of the yield strength is 1.13; tensile strength -1.10 ; elongation -1.84 . X-ray analysis of the texture helped understand how the crystallographic texture of the specimens changed under elongation in different directions. On the basis of strain-induced re-orientation of grains it was established that the active deformation mechanism included crystallographic slip of type $\{111\}<110\rangle$. Depending on the orientation of stress to the internal crystallographic axes, different texture components form as a result of elongation leading to the observed anisotropy of mechanical properties. To understand how changes in the crystallographic texture influence the mechanical properties of SLM products, a model has been developed for simulating anisotropy of mechanical properties on the basis of texture analysis data using DAMASK software. The input data include grain orientation, active deformation mechanism and parameters of the phenomenological law describing hardening of slip systems. A good correlation was found between the simulation data and the results of elongation obtained for experimental specimens printed at different orientation to the growth direction, in terms of both the yield strength anisotropy and the strain-induced changes in the crystallographic texture. Thus, knowing the difference in the crystallographic texture, the results of mechanical testing of standard shape specimens can be used to define the properties of specimens of a different shape.
\end{abstract}

\section{Introduction}

Additive manufacturing provides an advanced technique for making complex shape parts and is currently seeing an ever wider implementation in the production of steel structures [1-3]. Depending on the parameters of the selective laser melting (SLM) process (such as scan rate, laser power, scan path) and a part's geometry, different microstructure and crystallographic texture [4-7] can form, the combination of which will define the properties of the product [8]. While microstructure, i.e. the material structure and the presence of pores, influence the overall performance [9] of a part, anisotropy of physicomechanical properties is mainly governed by the type of crystallographic texture [10].

Parts of different geometry would have different optimal parameters of the SLM process leading to variations in grain structure and crystallographic texture. Because of this the mechanical properties obtained from standard shape specimens cannot be applied to specimens of a different shape (e.g. thin-walled parts, latticed parts, etc.). This, in turn, makes it more difficult to design parts with complex geometry to be produced by SLM. One possible solution of this problem can be establishing a relationship between the crystallographic texture and the anisotropy of mechanical properties of standard shape specimens made by SLM and further applying the results of simulation to complex shape parts. Thus, the texture analysis data on standard shape specimens designed for mechanical testing and on the complex shape part in view can be used to predict the anisotropy of mechanical properties using the results of crystallographic texture analysis. The aim of this research is to understand the relationship between the crystallographic texture and the anisotropy of mechanical properties, as well as to build and verify the accuracy of the model that could be used to simulate anisotropy in parts made by SLM from the austenitic steel powder 316L.

\section{Specimens and Methods Applied}

This paper examined SLM specimens made of austenitic steel 316L with the diameter of $4 \mathrm{~mm}$ and the length of $25 \mathrm{~mm}$. The following printing mode was applied: laser power $-357 \mathrm{~W}$, scan rate $-850 \mathrm{~mm} / \mathrm{sec}$., scan pitch $80 \mu \mathrm{m}$, powder layer thickness $-50 \mu \mathrm{m}$. Spherical powder was used with an average grain size of $40 \mu \mathrm{m}$. The SLM regime was selected to enable achieving maximum density of the material [11].

The specimens were printed so that the centre line of the specimen was at 0,45 or $90^{\circ}$ to the growth direction. $\mathrm{Z}$-axis indicates the layer growth direction, and $\mathrm{X}$ - and $\mathrm{Y}$-axes, which are perpendicular to each other, are in the plane perpendicular to $\mathrm{Z}$-axis and are equivalent to each other. The shape of the cylindrical specimens was standard for mechanical testing, and they were positioned differently to the growth direction Z: the centre line of Specimen 1 was parallel to $Z$-axis; the centre line of Specimen 2 was parallel to Y-axis; and the centre line of Specimen 3 was at $45^{\circ}$ to $\mathrm{Z}$ - and $\mathrm{X}$-axes. The centers of the SLM specimens were machined on a lathe for desired surface quality. 


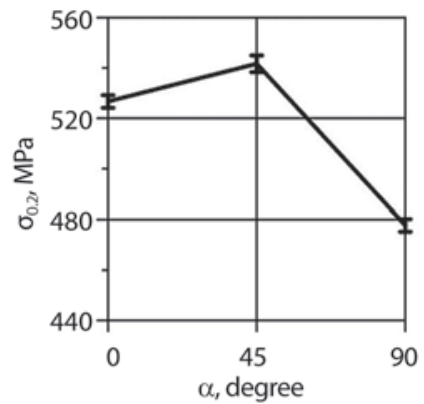

$\boldsymbol{a}$

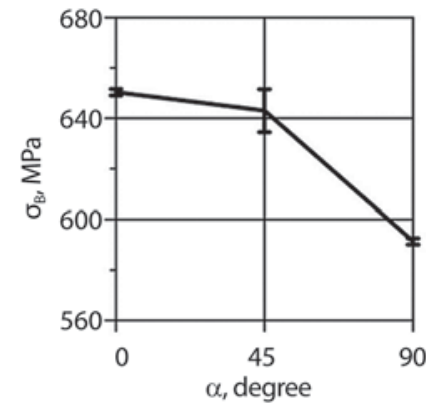

b



c

Fig. 1. Relationships between the mechanical properties and $\alpha$, i.e. the angle between the elongation and growth directions during printing: $a$ - yield strength; $b$ - tensile strength; $c$ - elongation

Mechanical tests were conducted at room temperature on a testing machine Instron 5982. Mechanical properties were analyzed based on stress-strain curves which were derived from the load-travel curves registered during the tensile test. The following properties were analyzed: yield strength, tensile strength, ultimate elongation. For this a series of measurements was conducted on three similar specimens and the obtained values were averaged out. Anisotropy of mechanical properties was analysed based on the ratio between the maximum and minimum values of a particular property.

To further analyze the relationship between the crystallographic texture and the material properties, one specimen was taken from each selection of specimens with the same orientation for X-ray analysis. The working section of the analyzed specimens was measured for reduction, on the basis of which the degree of strain was estimated in the cross section. Sections were sampled from each specimen that would have different strain degrees: $\varepsilon=0$, i.e. strain-free section; $\varepsilon=\varepsilon_{\max }$, i.e. section that is close to the fracture area; and $\varepsilon=\varepsilon_{\max } / 2$; and discshaped segments were cut out from them correspondingly. The segments were cut from longer halves of the fractured specimens.

The samples were cut on an EDM machine, which eliminates deformation. The surface studied was a cross section of the specimen perpendicular to its centre line. The standard surface preparation procedure was applied to prepare the sections of the specimens for X-ray analysis, i.e. the surface was polished and then subjected to electrolytic pickling in a solution of orthophosphoric and sulphuric acids and chromic anhydride.

The texture of the sections of all the studied specimens [12] was analyzed on an X-ray diffractometer DRON-3 equipped with an attachment for automatic texture analysis with filtered $\mathrm{K} \alpha$-radiation $\mathrm{Cr}$. The specimens were tilted "for reflection" and incomplete straight pole figures $\{111\},\{100\},\{110\}$ were registered, which were used for simulating an orientation distribution function (ODF) and rebuilding inverse pole figures (IPFs) for X, Y and $\mathrm{Z}$ directions.

\section{Experimental Results}

Fig. 1 shows the relationships between the mechanical properties of austenitic steel specimens and the $\alpha$ angle between the elongation direction (HP) and the growth direction $(Z)$ during printing.

It was found that the anisotropy of the yield strength is 1.13; tensile strength -1.10 ; elongation -1.84 . It means that the studied material has a significant anisotropy of mechanical properties.

Because the specimens had different orientations, the comparison was based on direct pole figures (DPFs) of the initial states of the specimens, which had been grown in different directions to the growth direction $\mathrm{Z}$. On the basis of the obtained results, it was established that when turned at the corresponding angle a the DPFs of all the studied specimens match.

Fig. 2 shows IPFs for the elongation direction of Specimens 1-3 for strain-free sections, near-neck sections and sections in between them. The grain re-orientation paths, which are indicated with arrows in the picture, can be established by analyzing sections having different degrees of strain. Analysis of the strain-induced re-orientation of the elongation axis was based on the changing pole density in the poles [[100]], [[110]] and [[111]] of the stereographic triangle.

\section{Model Description}

The deformation process was simulated with the help of DAMASK [13], which is a free software package allowing to monitor the changing orientation of grains induced by activation of possible crystallographic mechanisms of plastic deformation, i.e. to consider the crystalline structure of material at the grain level. At the basis of this modeling code lies a description of the global evolution in the quantitative distributions of temperatures or stresses with the help of static or dynamic equilibrium preservation laws. For instance, distributions of temperature fields or displacement fields can be calculated by analyzing the distribution of flows (heat, stresses), as well as the applied sources of heat and force. A 3-level model of the volume element is used for structural and multi-scale 


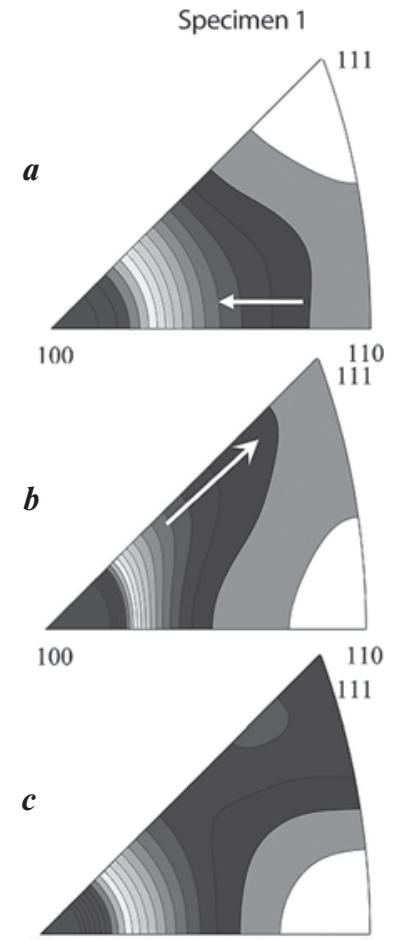

100



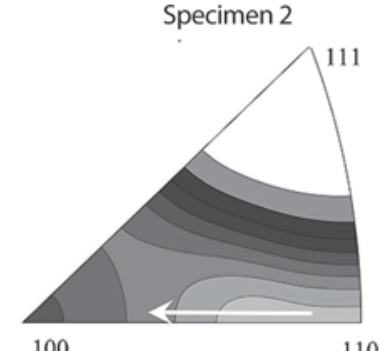

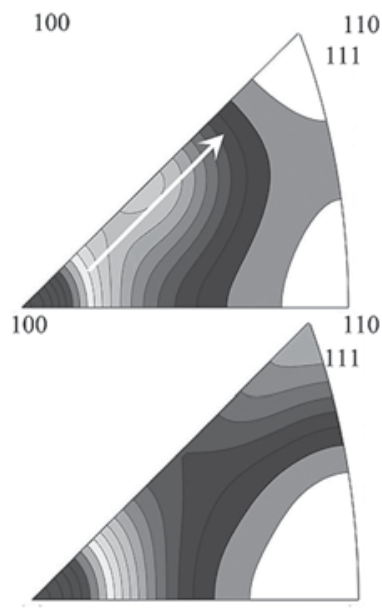

100

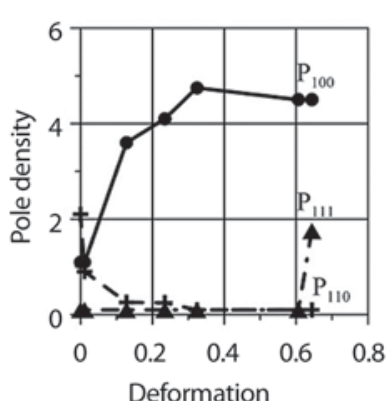

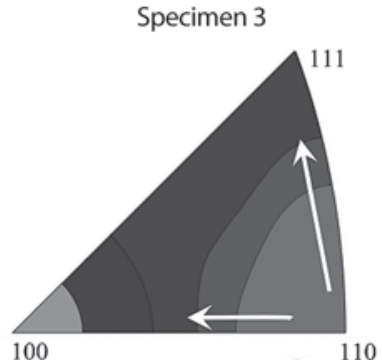

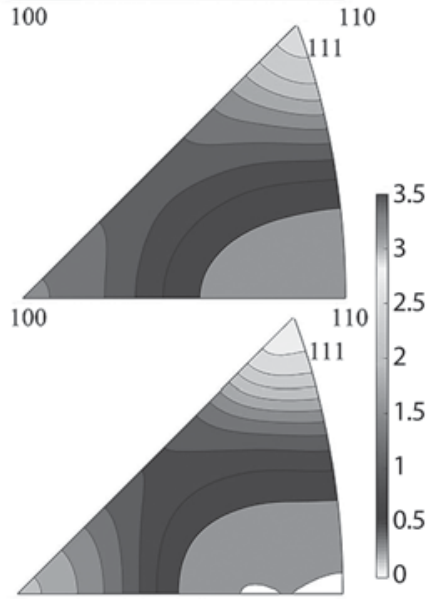

100

110

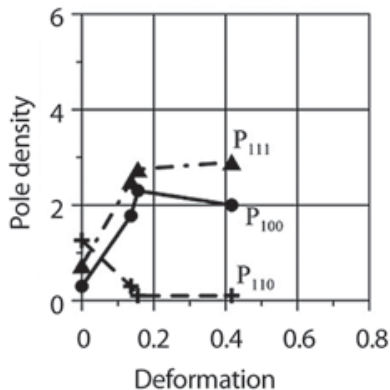

Fig. 2. Changing the ODF $(a-c)$ and pole density in different poles of the stereographic triangle $(d)$ to direct the elongation in Specimens 1-3 under growing strain: $a$ - strain-free section; $b$ - intermediate section; $c$ - neck. The arrows show the elongation axis re-orientation paths

description of flows and sources required by the conservation laws. At the highest level, the value of the target field in the element is determined for each element on the basis of microstructural components and the following homogenization. At the intermediate level, the reaction of each microstructural component can be determined through temporary integration of the main conservation laws applicable to flows and sources. At the lowest level, the reaction of the element can be determined with the help of state equations which rely on a set of variable state parameters that would correspond to the selected method for describing the material behaviour.

This research was based on a $10 \times 10 \times 10$ computational region, with each element constituting a grain with certain crystallographic orientation. Orientation of each element was chosen with the help of MTEX [14] on the basis of the experimental orientation distribution function (ODF) derived from the $\mathrm{X}$-ray texture analysis data.
Fig. 3, $\boldsymbol{a}$ shows the geometry of the applied model, the colour of the grains is related to the distribution of their Euler angle $\varphi_{1}$. Fig. 3, $\boldsymbol{b}$ shows the corresponding section of the ODF created on the basis of the selected set of discrete orientations with $\varphi_{2}=45^{\circ}$. As a criterion of proximity of this ODF and the experimental one (Fig. 3, c), we shall take a sum of mean square deviations of ODF in each point normalized to the total number of points. For the initial state of material this deviation is $L_{2}=0.16$.

A phenomenological model was applied to describe the relationship between the mechanical properties of the material and the degree of strain. For each deformation mechanism, such model uses a power dependence with the parameters selected on the basis of experimental data to define how the rate of deformation depends on the amount of strain accumulated in the element. Besides, the appropriate coefficients help account for the effect produced by different deformation mechanisms on each 

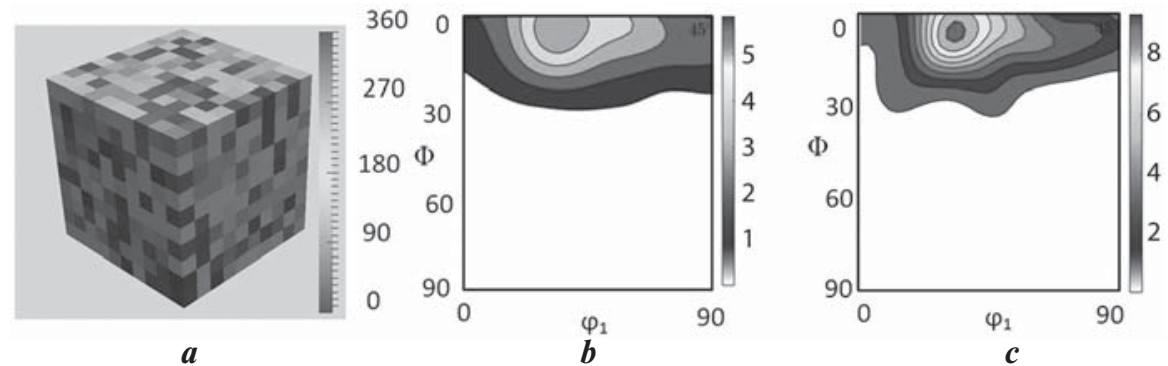

Fig. 3. Geometrical model of a polycrystal with grains with different Euler angle $\varphi_{1}$ orientation $(a)$, which corresponds to the ODF section with $\varphi_{2}=45^{\circ}$, with the ODF built on a set of selected orientations $(b)$ on the basis of experimental ODF $(c)$

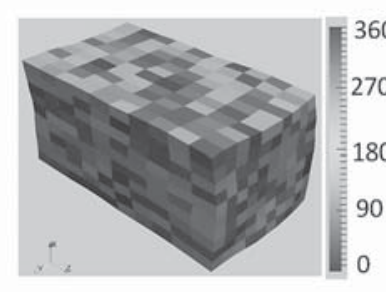

$\boldsymbol{a}$



$\boldsymbol{b}$

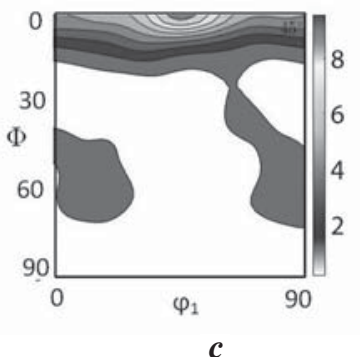

Fig. 4. Changes in the shape of the specimen and distribution of Euler angle $\varphi_{1}(a)$ and the ODF section $\varphi_{2}=45^{\circ}$, with the ODF built on the basis of simulation data (b) and experimental $(c)$ as a result of its elongation along the growth direction

other. A more detailed description of the model can be found in the paper [15]. The material in view was analyzed based on the crystallographic slip of type $\{111\}<110>$.

The values of the plasticity parameters applied in the model were selected for this material through variation and comparison of simulation data and experimental data obtained for standard shape specimens elongated along the growth direction.

\section{Results of Modeling}

Apart from simulating elongation along Z-axis to determine the model's plasticity parameters, the specimens were also elongated in the directions with $\alpha=45$ and $90^{\circ}$. The orientations of the elements used corresponded to the experimental data describing the crystallographic texture of the specimens which were arranged to have certain orientations during printing. After simulation, the average values of stresses and deformations were calculated for each step in time. Table 1 shows the measured and calculated values of the yield strength.

Changes in the crystallographic texture under plastic deformation serve as a sensitive tool indicative of the active mechanisms. Fig. 4, $\boldsymbol{a}$ shows the changing geometry

\begin{tabular}{|l|c|c|}
\hline \multicolumn{3}{|c|}{ Table 1. Yield strength st values as measured and calculated } \\
\hline \multicolumn{1}{|c|}{$\alpha$ angle, degrees } & $\sigma_{t}^{\mathrm{m}}, \mathrm{MPa}$ & $\sigma_{t}^{\text {calc }}, \mathrm{MPa}$ \\
\hline 0 & $542 \pm 4$ & 537 \\
\hline 45 & $527 \pm 3$ & 505 \\
\hline 90 & $478 \pm 6$ & 482 \\
\hline
\end{tabular}

of the simulated material and the grain distribution in Euler angle $\varphi_{1}$. Fig. 4, $\boldsymbol{b}$ shows an example of the ODF section that was built based on the simulation data for the specimen elongated in the growth direction. In this case the deviation of the ODF from the experimental one (Fig. 4, c) is $L_{2}=0.17$.

\section{Discussion}

Comparison of the elongation axis re-orientation paths observed in this study and indicated in Figure 2 with arrows and the paths predicted on the basis of Schmid factor distribution analysis in a stereographic triangle for the slip systems $\{111\}<110>$ and the trend for the slip direction toward the direction of elongation [16, 17], indicates that there is coherence between the two. Hence, the deformation of the FCC structure in the studied specimens can be attributed to the crystallographic slip that is consistent with the above mentioned system, i.e. $\{111\}<110\rangle$.

Moreover, comparison of Fig. 2, $\boldsymbol{c}$ and Fig. 2, $\boldsymbol{a}$ and $\boldsymbol{b}$ reveals that if the elongation axis is at $45^{\circ}$ to $Z$, the orientation of grains in which the planes $\{111\}$ are perpendicular to $\mathrm{Z}$ will be stabilized much sooner.

The curves in Fig. 2, $\boldsymbol{d}$ indicate that in Specimen 3 the stable orientation of the elongation axis along the normals $\{111\}$ can be reached when the degree of strain is $\approx 0.15$. This can explain the minimum plasticity of the material (see Fig. 1, c) when the application of load has this orientation. Greater hardening of the grains elongated under steady orientation is linked to the fact that the steady 
nature of orientation is maintained due to simultaneous and mutually coordinated action of a few slip systems. Whereas under unsteady orientation strain occurs as a result of slip of one or two systems, i.e. there is a weaker interaction between the active slip systems leading to weaker hardening.

Comparison of the ODF sections shown in Fig. 3 and 4 suggests that elongation is associated with uniformly changing crystallographic texture. The texture analysis data can help verify the choice of the deformation mechanism used in the model. Correlation between the simulated ODFs and experimental ODFs confirms that elongation of longitudinal specimens is associated with highly active crystallographic slip of the systems $\{111\}<110\rangle$. Some discrepancy between the experimental and simulation data may be attributed to inaccuracy when describing the experimental ODF (Fig. 3, c) with a set of discrete orientations (Fig. 3, b), as well as to the non-uniform strain near the neck of the deformed specimen. This is confirmed by the fact that the deviation of the ODFs built on the basis of the selected set of initial orientations and after simulation is quite close to the one of the corresponding experimental ODFs. This means that the active slip systems were identified correctly.

The yield strength values obtained for elongation in the directions with $\alpha=45$ and $90^{\circ}$ can be used to verify the anisotropy of the mechanical properties determined by the model, as the phenomenological parameters of the model were only determined based on the results of tensile tests involving elongation along the growth direction. Comparison of experimental and calculated yield strength values (see Table 1) indicates a good correlation between them.

\section{Conclusions}

1. It was found that the mechanical properties of the SLM specimens made of austenitic steel 316L have a significant anisotropy, which can be attributed to the sharp crystallographic texture found in the specimens.

2. A model was built for describing the plastic deformation of standard shape specimens. The model uses data on the material's crystallographic texture to simulate the mechanical properties of the studied specimens. The simulation data related to the specimens elongated in different directions to the growth direction are in good correlation with experimental findings.

3. The developed model can help predict how anisotropy of the mechanical properties of a part is likely to change depending on the crystallographic texture. Due to this the texture analysis data can be used to predict the mechanical properties of 3D printable complex shape parts.

\section{REFERENCES}

1. Guo N., Leu M. C. Additive manufacturing: technology, applications and research needs. Frontiers of Mechanical Engineering. 2013. No. 8. pp. 215-243.
2. Masaylo D. V., Popovich A. A., Orlov A. V., Gyulikhandanov E. L. Investigation of the structure and mechanical characteristics of specimens made by laser cladding and selective laser melting processes of spheroidized iron based powder. Chernye Metally. 2019. No. 4. pp. 73-77.

3. Petrovsky P. V., Cheverikin V. V., Sokolov P. Yu., Davidenko A. A. Dependence of the structure and properties of $03 \mathrm{Kh}$ $16 \mathrm{~N} 15 \mathrm{M} 3$ steel on the geometry of cellular structures obtained by the selective laser melting method. Chernye Metally. 2019. No. 3. pp. 49-53.

4. Choo H., Sham K.-L., John B., Austin Ng. et al. Effect of laser power on defect, texture, and microstructure of a laser powder bed fusion processed 316L stainless steel. Materials \& Design. 2019. No. 164. pp 1-12.

5. Alnajjar M., Christien F., Wolski K., Bosch C. Evidence of austenite by-passing in a stainless steel obtained from laser melting additive manufacturing. Additive Manufacturing. 2019. No. 25. pp. 187-195

6. Perclovich Yu. A., Isaenkova M. G., Dobrokhotov P. L, Rubanov A. E., Yudin A. V., Tretyako E. V. Nonuniformity of the Crystallographic Texture and the Mechanical Properties of the Austenitic Steel 316L Plates Formed by Selective Laser Melting of a Powder. Russian Metallurgy (Metally). 2019. No 1. pp. 42-47.

7. Isaenkova M. G., Perlovich Yu. A., Yudin A. V., Rubanov A. E. Formation of crystallographic texture in samples with different geometry from VT1-0 alloy, obtained by selective laser melting powder method. Tsvetnye Metally. 2018. No. 12. pp. 63-68.

8. Bahl S., Mishra S., Yazar K. U., Kola I. R. et al. Non-equilibrium microstructure, crystallographic texture and morphological texture synergistically result in unusual mechanical properties of 3D printed 316L stainless steel. Additive Manufacturing. 2019. No. 28. pp. 65-77.

9. Deev A. A., Kuznetcov P. A., Petrov S. N. Anisotropy of mechanical properties and its correlation with the structure of the stainless steel 316L produced by the SLM method. Physics Procedia. 2016. No. 83. pp. 789-769.

10. Kunze K., Etter T., Grässlin J., Shklover V. Texture, anisotropy in microstructure and mechanical properties of IN738LC alloy processed by selective laser melting (SLM). Materials Science \& Engineering A. 2015. No. 620. pp. 213-222.

11. Yudin A. V., Beregovsky V. V., Tretyakov E. V., Zhirnova J. E., Burmistrov M. A. Technological features manufacture samples of stainless steel 316L by Selective Laser Melting on the machine Melt Master3D-550. IOP Conference Series: Materials Science and Engineering. 2017. Vol. 218. No 1. pp. 12-22.

12. Perlovich Y., Isaenkova M., Fesenko V. Modern methods of experimental construction of texture complete direct pole figures by using X-ray data. IOP Conference Series: Materials Science and Engineering. 2016. No. 130. pp. 12-55.

13. DAMASK - the Düsseldorf Advanced Material Simulation Kit https://damask.mpie.de/Home/WebHome

14. MTEX v. 5.0.1 http://mtex-toolbox.github.io/

15. Roters F., Diehl M., Shanthraj P., Eisenlohr P. et al. DAMASK - The Düsseldorf Advanced Material Simulation Kit for modeling multi-physics crystal plasticity, thermal, and damage phenomena from the single crystal up to the component scale. Computational Materials Science. 2019. No. 158. pp. 420-478.

16. Calnan E. A., Clews C. J. B. Deformation textures in face-centered cubic metals. London, Edinburgh, and Dublin Philosophical Magazine and Journal of Science. 1950. No. 41(7). pp.1085-1100.

17. Vishnyakov Ya. D., Babareko A. A., Vladimirov S. A. et al. The theory of texture forming in metals and alloys. Moscow : Nauka, 1979. 344 p. 